\title{
Critical care admission for acute medical patients
}

\author{
Authors: Victoria Packham ${ }^{A}$ and Peter Hampshire ${ }^{B}$
}

Demand for intensive care is growing. There are no contemporaneous consensus guidelines on which patients should be referred to intensive care. Prognostic scoring systems predict survival, but are of limited use for individual patients. Some groups of patients have historically been regarded as having a very high mortality after admission to intensive care, raising questions about the appropriateness of advanced organ support in these patients. We reviewed the existing literature on outcomes of patients admitted to intensive care with chronic obstructive pulmonary disease, liver cirrhosis and haematological malignancies. We identified specific markers indicating a poor prognosis in each group, and also identified common risk factors predicting a high mortality across all groups. Multiple organ failure at the time of referral to intensive care predicts a very poor outcome. Physical factors indicating a limited functional capacity also predict high mortality, suggesting that frailty has a significant impact on intensive care outcome.

It is not medically appropriate to devote limited ICU resources to patients without reasonable prospect of significant recovery when patients who need those services, and who have a significant prospect of recovery from acutely life-threatening disease or injury are being turned away due to lack of capacity

- NIH Consensus Development Conference Statement, 1983

\section{Introduction}

Since the advent of intensive care, principles of triage have been used to develop admission criteria to intensive care units (ICUs) enabling organ support to be offered to patients with life-threatening organ failure, while avoiding harm from iatrogenic complications of inappropriate ICU admission. ${ }^{1}$ Prognostic scoring systems such as the Acute Physiology and Chronic Health Evaluation (APACHE II) predict hospital survival for large patient cohorts, but are not applicable to individuals. All clinicians should distinguish those patients who will benefit from escalation of care from those who are unlikely to benefit, in order to avoid inappropriately invasive

Authors: ${ }^{A}$ advanced clinical fellow in intensive care medicine, Intensive Care Unit, Royal Liverpool University Hospital, Liverpool, UK: ${ }^{\mathrm{B}}$ consultant in intensive care medicine, Intensive Care Unit, Royal Liverpool University Hospital, Liverpool, UK and burdensome treatments for patients with little survival potential. This approach reserves ICU admission for patients unlikely to survive without intervention, allowing patients whose disease process is so advanced to be managed in a conservative manner. However, there are no recent formal recommendations or consensus guidelines on which patients should be admitted to ICU. ${ }^{2}$

This article reviews the most recently reported outcomes for three specific patient groups for whom ICU admission has been considered controversial because of a perceived high mortality, identifying factors common to a poor prognosis, and discussing whether these are universally applicable to all patients.

\section{Chronic obstructive pulmonary disease}

Approximately 3.5 million people in the UK have a diagnosis of chronic obstructive pulmonary disease (COPD), and it is the fifth highest cause of death due to disease in the UK. Approximately $15 \%$ of patients die within three months of being admitted to hospital with an acute exacerbation, and median survival following an acute exacerbation requiring hospitalisation is 26 months. ${ }^{3,4}$

\section{Key points}

Identification of patients who will benefit from intensive care unit (ICU) admission is important to avoid inappropriate use of resources in an ageing population with multiple comorbidities.

Patients with chronic obstructive pulmonary disease (COPD) have a better prognosis than predicted by ICU clinicians after admission to ICU.

Severe functional limitation is an important predictor of mortality in patients with COPD.

Hospital mortality is $50-70 \%$ in patients with cirrhosis and haematological malignancies when admitted to ICU.

Frail patients and those who present in multiple organ failure are unlikely to benefit from admission to intensive care.

KEYWORDS: Intensive care, prognostic scoring systems, chronic obstructive pulmonary disease, cirrhosis, haematological malignancies, frailty 
Table 1. Studies investigating mortality of patients with COPD admitted to ICU.

\begin{tabular}{|c|c|c|c|c|c|}
\hline Author & Year & Patients, $n$ & ICU mortality, \% & Hospital mortality, \% & Long-term mortality, \% \\
\hline Hajizadeh $^{10}$ & 2015 & 4,791 & - & 23 & 12 months: 45 \\
\hline Wildman 5 (CAOS) & 2009 & 832 & 18.9 & 29.6 & 180 days: 37.9 \\
\hline Ucgun $^{6}$ & 2006 & 151 & - & 33.1 (52.9 if intubated) & - \\
\hline \multirow[t]{4}{*}{ Ai-Ping 4} & 2005 & 57 & - & 24.5 & 6 months: 39 \\
\hline & & & & & 1 year: 42.7 \\
\hline & & & & & 3 years: 61.2 \\
\hline & & & & & 5 years: 75 \\
\hline Wildman (ICNARC) ${ }^{9}$ & 2005 & 129,647 & 23.1 & 38.3 & \\
\hline \multirow[t]{4}{*}{ Breen $^{8}$} & 2002 & 74 & - & 20.3 & 6 months: 40.5 \\
\hline & & & & & 1 year: 48.6 \\
\hline & & & & & 2 year: 58.1 \\
\hline & & & & & 3 years: 63.5 \\
\hline
\end{tabular}

The COPD and Asthma Study (CAOS) identified that ICU clinicians are overly pessimistic when estimating mortality in patients with COPD. Intensivists predicted mean 180day survival at $49 \%$, when $62 \%$ of patients admitted to ICU survived to 180 days compared with $50 \%$ survival if not admitted to ICU. If decisions on whether to admit patients to critical care are based on pessimistic estimates of survival, some patients may be denied lifesaving ICU treatment. Furthermore, $73 \%$ of patient respondents considered their quality of life at 180 days the same or better than prior to ICU admission, and $96 \%$ respondents would choose similar treatment again. ${ }^{5}$ Several papers have reviewed outcomes for patients with COPD following admission to ICU (see Table 1).

The following parameters are consistent independent risk factors for in-hospital and short-term mortality: ${ }^{6-8}$

$>$ APACHE II score

$>$ invasive mechanical ventilation (IMV)

$>$ low Glasgow Coma Score (GCS) on presentation to ICU

$>$ non-respiratory organ failure

> right ventricular failure or arrhythmias prior to ICU admission

> increasing mortality with increasing number of organs failing (respiratory failure with further three organs failing (in-hospital mortality $83.3 \%$ ))

$>$ poor baseline nutrition (hypoalbuminaemia or body mass index $<20$ )

$>$ severe baseline disease; $\mathrm{FEV}_{1}<0.74 \mathrm{~L}$

$>$ cardiopulmonary resuscitation $<24$ hours prior to admission $>$ advanced age.

The following variables have also been identified as significant risk factors for 180 -day mortality: ${ }^{9}$

$>$ age $>70$ years, with progressively higher risk for the age groups $>75$ and $>80$ years

$>$ male gender

$>$ duration of hospital admission prior to ICU admission (with lowest risk if admission is immediate, and highest risk found between days 4-7)
$>$ physical functional limitations (housebound $44.3 \%$ mortality; bed/chair bound 64.9\%)

$>$ mid-upper arm circumference $<20 \mathrm{~cm}$

$>$ atrial fibrillation (on presentation)

$>$ APACHE II score

$>$ low GCS at presentation to ICU.

The introduction of non-invasive ventilation in separate respiratory units could explain the reduction in ICU mortality, as some potentially high-risk patients will not be referred.

It would follow from the low in-hospital mortality rates that the implications for clinicians are: a full and frank discussion with the patient and family regarding prognosis in the event of an acute exacerbation, including such factors as quality of life, the likelihood of increased dependency in $60 \%$ patients and possibility of discharge to a higher care facility. ${ }^{10}$

\section{Liver cirrhosis}

A $160 \%$ increase in hospital admissions for cirrhosis from 1983-1995, was mirrored by a $160 \%$ increase in hospital admissions with alcoholic liver disease (ALD) from 1996-2000. Admissions to ICUs in England and Wales of patients with chronic liver disease and ALD have tripled in number. ${ }^{11}$ Several studies have reviewed outcomes following ICU admission in patients with cirrhosis (see Table 2).

Factors associated with better prognosis for long-term survival include variceal bleeding and admission to ICU. ${ }^{12,13}$ Factors associated with poor short-term prognosis include first presentation, ascites, hyperlactataemia, sepsis and septic shock. ${ }^{14}$ Longer term poor outlook is associated with hepatic encephalopathy alone, which has only a $20 \%$ survival rate at one year. ${ }^{12}$

Discriminatory models of mortality prediction can guide clinical decision-making. ${ }^{15}$ The Model for End-stage Liver Disease (MELD) score, Royal Free model and Child-Pugh (CPS) are well validated for cirrhosis. ICU-specific scoring systems, such as APACHE II, Simplified Acute Physiology Score 
Table 2. Studies reviewing mortality rates in the ICU population with cirrhosis.

\begin{tabular}{llllll}
\hline Study & Year & Patients, $\mathbf{n}$ & ICU mortality, \% & Hospital mortality, \% & Long-term mortality, \% \\
Mackle $^{12}$ & 2006 & 107 & 58 & 71 & $81 \%$ (1 year) \\
Welch $^{11}$ & 2008 & 4,219 & 49.4 & 67.1 \\
Cholangitis $^{15}$ & 2009 & 312 & - & 65 \\
McPhail $^{19}$ & 2012 & 958 & 63 & 51 \\
Beejooa $^{14, a}$ & 2013 & & - & 49 \\
Theocharidou & & & - & 52.3 \\
\hline atertiary, non-transplant settings. ICU = intensive care unit.
\end{tabular}

(SAPS) and Sequential Organ Failure Assessment (SOFA) scores discriminate well between survivors and non-survivors (AUROC $>0.8) .{ }^{13}$ Scores of APACHE II $>30$, SAPS II $>60$, SOFA $>12$, CPS $>12$ are all associated with mortality of $>90 \%$. The MELD score is an accurate predictor of death within three months following variceal bleeds. ${ }^{16}$ A MELD score on presentation of $>25$ confers a 7 -day mortality rate of $28.3 \%$ and 30 -day mortality of $53.6 \% .{ }^{17}$

Organ failure on a background of cirrhosis is consistently associated with poor prognosis, with over $90 \%$ mortality in patients with three or more organ failures. The following are independent predictors of mortality: ${ }^{17}$

$>$ IMV in association with MELD score $\geq 25$

$>$ vasopressor requirement

$>$ SOFA scores at day $1 \geq 17$ and day $3 \geq 12$ have a $100 \%$ positive predictive value for mortality. ${ }^{18}$

McPhail et al demonstrated improvement in survival rates from $47 \%$ to $73 \%$ in patients admitted to ICUs in the UK with chronic liver disease over 10 years. This was attributed to improvements in ICU management, and fewer patients admitted with renal and three organ failure, indicating improvement in prognostication and identification of endof-life issues. ${ }^{19}$ Escalation of cirrhotic patients with sepsis requiring more than two organ support is unlikely to result in survival. The European Association for the Study of the Liver recommends consideration of organ support for patients with a 'pre-morbid MELD $<15$, questioned if MELD is $>30$ and there is three or more organ failure'. ${ }^{20}$ The authors agree with these recommendations. Lack of response to organ support after 48 hours should prompt reconsideration of the benefits of continued treatment in patients who are admitted to ICU. The implication for physicians from the outcome data discussed above is that patients with liver cirrhosis and multiple organ failure are extremely unlikely to benefit from escalation of treatment.

\section{Haematological malignancies}

The management of patients with haematological malignancies (HM) has improved dramatically over the last 20 years. Patients with $\mathrm{HM}$ often undergo treatments placing them at increased risk of infection and can rapidly deteriorate into multiple organ dysfunction syndrome (MODS). Improved chemotherapy and biological adjuvant agents has improved survival rates for some types of HM, but five-year survival rates for Hodgkin's disease and myeloma remain unchanged. ${ }^{21} \mathrm{~A}$ multicentre study by Hampshire et al ${ }^{22}$ included 7,689 patients with HM. In total, $43.1 \%$ of patients died in ICU, and 59.2\% died in hospital. Hospital mortality was associated with the number of organ failures; $98.8 \%$ mortality was observed with five organ failures. More recent studies demonstrate improving survival in patients with $\mathrm{HM}$, with hospital survival rates of 54-60\%, and 12-month survival of $43 \%$. $^{23,24}$ Similar improvements in survival have been documented in patients receiving haemopoietic stem cell transplants (HSCT). ${ }^{25}$ This improvement in survival has been attributed to better selection of appropriate patients, treatment in specialist centres and advances in treatment of organ failure. ${ }^{26}$

Neutropenia confers little increased mortality risk. ${ }^{22}$ Factors consistently predicting a poor outcome in patients admitted to the ICU with HM are:

$>\mathrm{IMV}$

$>$ MODS

> increasing prognostic score (such as APACHE II, SAPS II or Intensive Care National Audit \& Research Centre (ICNARC) score).

Severity of acute illness has greater influence on the outcome of ICU admission than factors related to the malignancy. A trial of full intensive care support for a limited time (up to five days) allowing assessment of response to treatment for patients with HM is recommended. However, patients who are not expected to survive six months, who have undergone repeated HSCT or those already in established MODS, should not be admitted as ICU admission will not improve their chance of survival. For clinicians, the implication from this data is that early referral of critically ill patients with HM is warranted, since they can deteriorate rapidly and their outcomes have significantly improved.

\section{Conclusion}

Advances in medical care over the last 50 years have resulted in increased life expectancy, but also the prevalence of significant comorbidities. Recent data suggest that certain conditions deemed unsurvivable, are now not so. An increase in hospital and ICU admissions, and high bed occupancy necessitate appropriate resource allocation. We chose three specific patient groups to examine the factors that determine outcomes when admitted to ICU. In all three patients groups discussed, when MODS is established, ICU 
admission achieves little to alter the prognosis. Traditional scores predicting ICU outcome rely heavily on measures of acute physiological derangement, but do not incorporate any significant measures of prehospital functional status or frailty. No existing studies have prospectively evaluated the influence of frailty on outcomes in critically ill patients, however there is increasing recognition that frailty has a significant impact on intensive care. ${ }^{27}$ Patients exhibiting signs associated with frailty - in particular malnutrition and poor functional status - have a higher mortality, indicating a lack of capacity to cope with and then recover from the demands of ICU treatment. Instead, an honest conversation between physicians, patients and their families regarding treatment options and limitations of treatment should be encouraged.

\section{References}

1 NIH Consensus Statement. Crit Care Med 1983 Mar 7-9;46:1-26.

2 Rubenfeld G. Guidelines for ICU Admission, Discharge and Triage. Crit Care Med 1999;27:633-8.

3 Department of Health. Facts about COPD [GR:11943]. London: DH, 2010. Available online at http://webarchive.nationalarchives. gov.uk/+/www.dh.gov.uk/en/Healthcare/Longtermconditions/ COPD/DH_113006 [Accessed 8 June 2015].

4 Ai-Ping C, Lee KH, Lim TK. In-hospital and 5-year mortality of patients treated in ICU for acute exacerbation of COPD. Chest 2005;128:518-24.

5 Wildman MJ, Sanderson CF, Groves J et al. Survival and quality of life for patients with COPD or asthma admitted to intensive care in a UK multi-centre cohort: the COPD and Asthma Outcome Study (CAOS). Thorax 2009; 64:128-32.

6 Ucgun I, Metintas M, Moral H et al. Predictors of hospital outcome and intubation in COPD patients admitted to the respiratory ICU for acute hypercapnic respiratory failure. Resp Med 2006;100:66-74.

7 Wildman MJ, Harrison DA, Brady AR et al. Case mix and outcomes for admissions to UK adult, general critical care units with chronic obstructive pulmonary disease: a secondary analysis of the ICNARC Case Mix Programme Database. Crit Care 2005;9(Suppl 3):S38-48.

8 Breen D, Churches T, Hawker F, Torzillo PJ. Acute respiratory failure secondary to chronic obstructive pulmonary disease treated in the intensive care unit: a long term follow up study. Thorax 2002;57:29-33.

9 Wildman MJ, Sanderson C, Groves J et al. Predicting mortality for patients with exacerbations of COPD and asthma in COPD and Asthma Outcome Study (CAOS). QJM 2009;102:389-99.

10 Hajizadeh N. What happens to patients with COPD with long-term oxygen treatment who receive mechanical ventilation for COPD exacerbation? A 1-year retrospective follow-up study. Thorax 2015;70:294-6.

11 Welch C, Harrison D, Short A, Rowan K. The increasing burden of alcoholic liver disease on United Kingdom critical care units: secondary analysis of a high quality clinical database. J Health Serv Res Policy 2008;13(Suppl 2):40-4.

12 Mackle IJ, Swann DG, Cook B. One year outcome of intensive care patients with decompensated alcoholic liver disease. Br J Anaesth 2006; 97:496-8.
13 Theocharidou E, Pieri G, Mohammad AO et al. The Royal Free Hospital Score: A calibrated prognostic model for patients with cirrhosis admitted to intensive care unit. Comparison with current models and CLIF-SOFA score. Am J Gastroenterol 2014;109:554-62.

14 Beejooa G, Oates R, Loo L et al. PTU-105 Outcome of cirrhotic patients admitted to intensive care units at hospitals without specialist liver services. Gut 2013;62:A89.

15 Cholongitas E, Senzolo M, Patch D et al. Risk factors, sequential organ failure assessment and model for end-stage liver disease scores for predicting short term mortality in cirrhotic patients admitted to intensive care unit. Aliment Phamacol Ther 2006;23:883-93.

16 Kamath PS, Wiesner RH, Malinchoc M et al. A model to predict survival in patients with end-stage liver disease. Hepatology 2001;33:464-70.

17 Bahirwani R, Ghabril M, Forde KA et al. Factors that predict short-term intensive care unit mortality in patients with cirrhosis. Clin Gastroenterol Hepatol 2013;11:1194-200.

18 Das V, Boelle PY, Galbois A et al. Cirrhotic patients in the medical intensive care unit: early prognosis and long term survival. Crit Care Med 2010;38:2108-16.

19 McPhail MJ, Shawcross DL, Abeles RD et al. Changing outcomes in patients with chronic liver disease in intensive care: a decade of experience. Crit Care 2012;16(Suppl 1):P393.

20 EASL. EASL Clinical Practice Guidelines: management of alcoholic liver disease. J Hepatol 2012;57:399-420.

21 Brenner H, Gondos A, Arndt V. Recent major progress in long-term cancer patient survival disclosed by modeled period analysis. J Clin Oncol 2007;25:3274-80.

22 Hampshire P, Welch CA, McCrossan LA et al. Admission factors with hospital mortality in patients with haematological malignancy admitted to UK adult, general critical care units: a secondary analysis of the ICNARC Case Mix Programme Database. Crit Care 2009;13:R137.

23 Azoulay E, Mokart D, Pene F et al. Outcomes of critically ill patients with hematologic malignancies: prospective multicenter data from France and Belgium - a groupe de recherche respiratoire en réanimation onco-hématologique study. J Clin Oncol 2013;31:2810-8.

24 Bird GT, Farquahar-Smith P, Wigmore T et al. Outcomes and prognostic factors in patients with haematological malignancy admitted to a specialist cancer intensive care unit: a 5 yr study. Br J Anaesth 2012;108:452-9.

25 Pène F, Aubron C, Azoulay E et al. Outcome of critically ill allogeneic hematopoietic stem-cell transplantation recipients: a reappraisal of indications for organ failure supports. J Clin Oncol 2006;24:643-9.

26 Hampshire PA, Pugh R, Hajimichael P. Outcomes for critically ill patients with haematological malignancies in specialist and nonspecialist centres in the United Kingdom. J Cancer Ther Res 2014:3;5.

27 McDermid RC, Stelfox HT, Bagshaw SM. Frailty in the critically ill: a novel concept. Crit Care 2011;15:301-6.

Address for correspondence: Dr P Hampshire, Intensive Care Unit, Royal Liverpool University Hospital, Prescot Street, Liverpool L7 8XP, UK.

Email: peter.hampshire@rlbuht.nhs.uk 